\title{
Erratum to: A bedr way of genomic interval processing
}

Syed Haider ${ }^{1 \dagger}$, Daryl Waggott ${ }^{1 \dagger}$, Emilie Lalonde ${ }^{1,2}$, Clement Fung ${ }^{1}$, Fei-Fei Liu ${ }^{2,3}$ and Paul C. Boutros ${ }^{1,2^{*}}$

\section{Erratum}

In the HTML version of this article that was originally published [1] the Venn diagram in the section titled "Visualization" was missing and was replaced with source code. Please see the missing Venn diagram below:

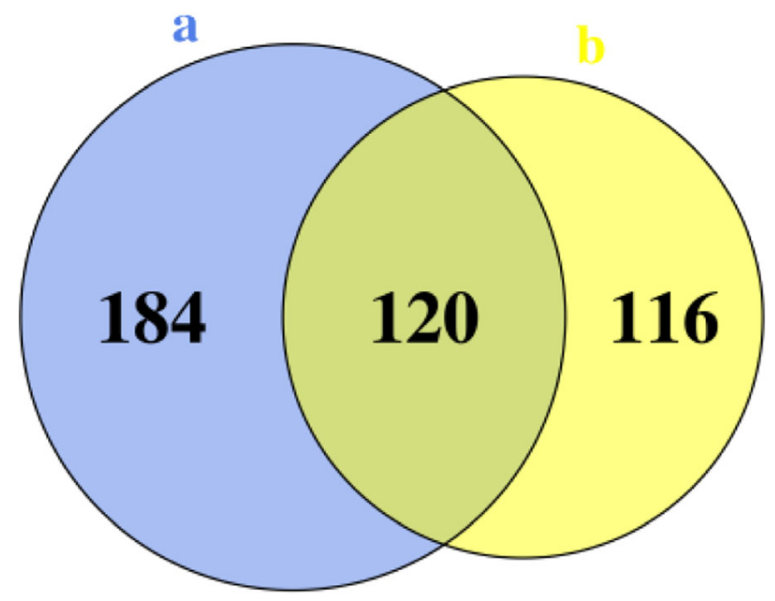

The original article has been revised and the publisher apologises for the error.

\section{Author details}

${ }^{1}$ Informatics and Biocomputing Platform, Ontario Institute for Cancer Research, Toronto M5G 0A3, Canada. Departments of Radiation Oncology, Pharmacology \& Toxicology, and Medical Biophysics, University of Toronto, Toronto M5G 2M9, Canada. ${ }^{3}$ Ontario Cancer Institute and Campbell Family Institute for Cancer Research, Princess Margaret Hospital, University Health Network, Toronto M5G 2M9, Canada.

Received: 22 December 2016 Accepted: 22 December 2016 Published online: 09 January 2017

\section{Reference}

1. Haider S, Waggott D, Lalonde E, Fung C, Liu F, Boutros PC. A bedr way of genomic interval processing. Source Code Biol Med. 2016;11:14.

\footnotetext{
* Correspondence: Paul.Boutros@oicr.on.ca

${ }^{\dagger}$ Equal contributors

${ }^{1}$ Informatics and Biocomputing Platform, Ontario Institute for Cancer Research, Toronto M5G 0A3, Canada

${ }^{2}$ Departments of Radiation Oncology, Pharmacology \& Toxicology, and

Medical Biophysics, University of Toronto, Toronto M5G 2M9, Canada
} 